\title{
El evento raro. Epistemología y complejidad
}

\author{
THE RARE EVENT. EPISTEMOLOGY AND COMPLEXITY
}

Carlos Eduardo Maldonado (carlos.maldonado@urosario.edu.co) Facultad de Ciencia Política y Relaciones Internacionales, Universidad del Rosario (Bogotá, Colombia) ORCID: 0000-0002-9262-8879

\begin{abstract}
Rare events have happened ever since the beginning of this universe. However, they have very recently become the subject of study and a category. This paper studies what rare events are and explores a series of epistemological consequences, thereafter, along with its complexity. The paper introduces a brand new understanding about the sciences of complexity, namely the study of unpredictable phenomena. Unpredictability is the ground, the problem, and the support for complexity as such, something that has never been openly set to the fore in the bibliography about the field.
\end{abstract}

Key words: rare events, sciences of complexity, theory of knowledge, science, philosophy.

\section{Resumen}

Los eventos raros han sucedido desde el comienzo de este universo y, sin embargo, la tematización y categorización de los mismos es un fenómeno perfectamente reciente. Este artículo estudia qué son los eventos raros y explora la tesis y consecuencia de tipo epistemológico y su complejidad. El texto permite por lo demás una nueva comprensión sobre las ciencias de la complejidad, a saber, el estudio de fenómenos impredecibles. La impredecibilidad constituye el piso, el problema y el soporte de la complejidad misma, algo que jamás ha sido puesto explícitamente de manifiesto en la bibliografía sobre el tema.

Palabras clave: eventos raros, ciencias de la complejidad, teoría del conocimiento, ciencia, filosofía.

\section{Introducción}

En contraste con lo que enseñan las ciencias fisicalistas, el mundo y la naturaleza no están hechos de fenómenos universales, generales y comunes. Por el contrario, la vida de los seres humanos y de la naturaleza está constituida por acontecimientos extraordinarios, excepcionales, cuando dichos acontecimientos son significativos. La vida está constituida por innumerables acontecimientos excepcionales. Únicos, literalmente. Una aproximación (bastante desafortunada) consiste en decir que la existencia se define por oportunidades.

De otra parte, la tierra parece ser un lugar único, la persona a la que amamos es diferente a cualquier otra, hemos tenido experiencias que a cada quien le parecen singulares e irrepetibles, y así sucesivamente. $Y$ sin embargo existen los días anónimos, gente que nos es indiferente, lugares que no nos significan particularmente nada. 
Pues bien, la complejidad del mundo consiste justamente en la excepcionalidad, el carácter extraordinario, en fin, irreversible de las experiencias, las vivencias, los acontecimientos.

Desde luego que tratamos de realizar generalizaciones, como formas de elaborar clases y conjuntos de fenómenos, o llevar a cabo patrones. El salto a la generalización es un problema que ya ha sido identificado hace tiempo como una patología de la razón. Y patologías las hay más graves y más ligeras. Se trata, notablemente, del problema de la inducción-que Popper declaró haber resuelto definitivamente.

Vivimos un mundo moteado, con vetas. No uniforme ni estándar o lineal. Vetas cromáticas, vetas geológicas, vetas sonoras, por ejemplo. Es lo que puede traducirse como un mundo con escalas, gradientes, modos, dimensiones diversas. La verdad es que el mundo y la naturaleza son irregulares. Pero si es así hay que hacer una ciencia de dicha irregularidad.

Son las irregularidades las que dan qué pensar. Son ellas en las que el conocimiento se estrella contra lovisto, lo-conocido, lo-experienciado. Sin las excepciones el pensamiento se abandona a sí mismo y se convierte en un comportamiento o en fenómeno físico, sin más. Es entonces cuando nos devoran los hábitos y las costumbres, en fin, los automatismos, que son las simientes de un mundo conservador y autorreferente. En fin, la ciencia, como la vida, está colmada de eventos raros.

Los eventos raros deben poder ser comprendidos y explicados, y esa exigencia, al mismo tiempo moral, existencia y teórica, es la que sienta las bases para una ciencia de lo singular y extraordinario. Históricamente y culturalmente hablando nos encontramos apenas en los umbrales de dicha ciencia. Este artículo se da a una dúplice tarea: de un lado, explicar qué es un evento raro; y sobre esta base, de consuno, hacer explícitas las relaciones entre epistemología y complejidad. Es sorprendente que la bibliografía sobre ambos aspectos no sea precisamente abundante.

Cinco argumentos sostienen este texto. En el primero se muestra la emergencia del problema de los eventos raros en la reflexión científica. El segundo pone de manifiesto la crisis de la ciencia normal, debido justamente al descubrimiento de eventos raros. El tercer argumento se concentra en la complejidad de los eventos raros y suministra una comprensión diferente de las ciencias de la complejidad. El sexto hace explícito en qué consiste exactamente un evento raro y, sobre esta base, avanza al último argumento que sienta las bases epistemológicas para entender los eventos raros y trabajar y vivir con ellos. Al final se extraen algunas conclusiones.

\section{Emergencia de eventos raros}

De partida, cabe hacer una breve anatomía que permite, al mismo tiempo, trazar los orígenes de los eventos raros como problema científico, filosófico y práctico.

Exactamente en el polo opuesto del determinismo se encuentran los eventos raros. Justamente, aquellos que rompen esquemas y patrones, que son por definición impredecibles, en fin, acontecimientos sorpresivos. Esta clase de eventos fueron genéricamente tratados en la historia como milagros, casualidades, azares de la fortuna, y demás. Pero es tan solo recientemente cuando adquieren una categorización propia, en particular a partir de los desarrollos de la computación y el desarrollo de la modelación de eventos raros (REM en inglés: rare events modeling). Los desarrollos de la estadística han sido igualmente determinantes al respecto, gracias a lo cual cabe destacar eventos extremos, un concepto sobre el cual volveremos en la sección siguiente. Puede decirse que es propiamente en los años 1990s 
cuando el concepto, la categoría, y técnicas concomitantes para el estudio de los eventos raros fueron desarrollados con toda propiedad.

Ya en el marco de la teoría de la decisión racional el tema se toca, analíticamente, por dos frentes distintos: de un lado, con las decisiones que se toman en condiciones de riesgo, y de otra parte al mismo tiempo, con la teoría de la utilidad esperada. Las primeras, sobre la base de las incertidumbres, enfocan los problemas de racionalidad, de percepción del riesgo y las consecuencias que se derivan. Por su parte, la teoría de la utilidad esperada pone de manifiesto la valoración que los sujetos asignan a sus propias acciones y a su propia vida: nadie hace nada de lo que no espera obtener un resultado mayor que si dejara de hacerlo.

Por su parte, del lado de las ciencias de la complejidad, los eventos raros son antecedidos como comportamientos caóticos, las catástrofes y el estudio de la sorpresa. Sin embargo, en honor a la verdad, se trata de cosas que en el espíritu apuntan en una misma dirección, pero que en la letra son totalmente distintas. En efecto, el caso es determinista, la teoría de catástrofes es un lenguaje que permite estudiar cambios súbitos, imprevistos e irreversibles, y el tema de las sorpresas -por ejemplo las emergencias-, no llega a ser nunca más que una aproximación a la comprensión de un fenómeno o sistema.

De manera generalizada, en el marco del estudio de los eventos raros es común leer en la bibliografía especializada acerca de eventos catastróficos que tienen perturbaciones a corto plazo y cambios a largo plazo. La cultura científica y social que hace este descubrimiento coincide exactamente con el final del siglo XX y se proyecta hasta nuestros días. Se trata, en efecto, de una época que observa cambios súbitos, imprevistos e irreversibles fenómenos poco usuales y conocidos y que se da entonces a la tarea de explicarlos y comprenderlos. Al fin y al cabo, cada época desarrolla la ciencia que puede y la ciencia que necesita. Y la de nuestros días, todo parece indicarlo, es ciencia de eventos raros; no de fenómenos regulares, sujetos a grandes números, y para los cuales la estadística clásica era básicamente suficiente.

Culturalmente, la preocupación por los eventos raros coincide con el tránsito del capitalismo postindustrial a la sociedad de la información, y desde ésta hasta la sociedad del conocimiento y la sociedad de redes (desde otro punto de vista, los eventos raros son epistemológicamente contemporáneos, grosso modo, con la emergencia de la ciencia de grandes datos [big-data science] y las grandes bases de datos [big data]). En hidrología y en meteorología, en ingeniería y en política, en economía y en finanzas, incluso en ámbitos de la salud (epidemiología) y de los sistemas informáticos, por ejemplo, los eventos raros son fenómenos de estudio a fin de prevenirlos puesto que los costos en numerosos ámbitos pueden ser elevados. Originariamente, los eventos raros permanecen atados al estudio de los riesgos $y$, desde este punto de vista, los enfoques bayesianos predominan durante un largo período en el estudio y comprensión de los eventos extremos. Un evento se dice extremo precisamente porque se encuentra en los extremos de una curva gaussiana o de distribuciones normales.

La estadística introduce un criterio fundamental en la lectura del universo y la realidad, a saber: comprendemos los fenómenos, los comportamientos y los sistemas en términos de distribuciones, y consiguientemente, de probabilidades de ocurrencia. Las dinámicas de estas probabilidades de ocurrencia constituyen el objeto del cálculo. De esta suerte, estadística y cálculo son las herramientas mediante las cuales se comprende el mundo y la sociedad, e históricamente hablando, esta es la característica epistemológica de lejos más importante de la burguesía como clase social. 
Ahora bien, la ciencia clásica siempre supo de los extremos de una distribución normal, pero siempre los descartó con diferentes argumentos, tales como: parametrización, desviaciones estándares, razonamientos por defectos, y otros. Consiguientemente, el mundo y la realidad siempre fueron comprendidos y explicados justamente en términos de estándares, medias, medianas, umbrales, promedios, matrices, y demás. La tragedia científica, filosófica y práctica estriba justamente en el reconocimiento de que aquellos espectros y umbrales que la ciencia normal no consideró aunque supiera sobre ellas, fueron las que, sorpresivamente se convirtieron al cabo en los más significativos. Ello condujo y ha conducido, sistemáticamente, a una crisis de la ciencia normal.

\section{Crisis de la ciencia normal}

Históricamente hablando, la burguesía es la primera clase social que ve el movimiento y para explicarlo desarrolla dos herramientas fundamentales: la estadística (Grünewald) y el cálculo (integral e infinitesimal) (Leibniz - Newton). Gracias a ellas, la burguesía logra explicar el universo y la sociedad en términos de movimientos cíclicos, periódicos, regulares. Todo lo demás, hasta la fecha, es todo lo de menos: esto es, a lo sumo las discusiones se centran en si se trata de ciclos más amplios o más estrechos -de producción, de crisis, etc. Este es exactamente el mindset de la modernidad y de la burguesía como clase social en la historia.

La estadística en general -esto es, distribuciones uniformes, de Bernouilli, geométrica e hipergeométrica, binomial y negativa binomial, de Poisson, exponencial, gaussiana, chi-cuadrado, gama, beta y otras-, y la bayesiana en particular (por ejemplo las redes bayesianas) han sido ampliamente empleadas en el estudio de los eventos raros. Sin embargo, existe un reconocimiento explícito en buena parte de la bibliografía acerca de las insuficiencias de estas aproximaciones.

Podemos denominar a las distribuciones estadísticas anteriores como estadística normal. Frente a ellas cabe destacar dos tipos de distribuciones que no son "normales" y que, por el contrario, conducen en dirección a la complejidad o a los eventos raros, dos modos distintos de designar un solo y mismo tema. Estos dos tipos son: las leyes de potencia y la teoría del valor extremo.

En verdad, las leyes de potencia, formuladas originariamente por Zipf, fueron re-descubiertas gracias a los trabajos de Mandelbrot, y fueron definitivamente incorporadas en el estudio de la complejidad a partir de los trabajos de Per Bak y su grupo, notablemente con la incorporación de la criticalidad auto-organizada. De otra parte, independientemente, anticipados por L. Tippett y R. A. Fischer, E. J. Gumbel desarrolla a partir de los años 1950s-60s, una teoría de valores extremos. Estos valores extremos son genéricamente conocidos también como las distribuciones de colas largas, y se concentran en desviaciones extremas en las distribuciones de un fenómeno determinado.

En cualquiera de los dos casos, resulta manifiesto que el mundo ni consiste ni puede ser explicado en términos de estándares, promedios, medias, medianas y demás. Concomitantemente, traducido al lenguaje de la ciencia política este reconocimiento implica la crisis total de las políticas públicas, puesto que, por definición, las políticas públicas son políticas para las mayorías, en términos justamente de una curva de Bell. A título conjetural, cabe pensar que, análogamente a como se produce en la ciencia de punta un giro de la medicina epidemiológica hacia la medicina personalizada, asimismo debe ser posible una política estatal o gubernamental que atienda a los grupos minoritarios, marginados o anormales de la sociedad como foco central; esto es, la existencia de políticas diferenciadas de sociedad. Decíamos, en una palabra, el mundo no consiste, ni se funda en la ley de grandes números. Por tanto, la idea del mundo y la 
naturaleza fundada esencialmente en ciclos -más amplios o restringidos-; esto es, en movimientos y dinámicas regulares y periódicas es o bien un mecanismo de simplificación, o bien, sencillamente, un error.

Digámoslo a título de provocación: los movimientos cíclicos, periódicos, regulares son, en realidad, excepciones frente a la norma, que son cambios súbitos, imprevistos, irreversibles. Solo que culturalmente hablando jamás habíamos hecho de estos últimos el objeto explícito de tematización y problematización. La complejidad ex ante no existe ni existió; cada época logra los descubrimientos del mundo, la naturaleza y la sociedad que puede llevar a cabo y con ellos, retrospectivamente, enriquece a la historia (anterior).

Pues bien, decía M. Castells que en el marco de la sociedad de la información emerge una nueva clase social que no tiene la propiedad sobre los medios de producción y que no necesita tenerla, pero que es la productora de riqueza y bienestar en esta nueva fase de la familia humana. Extrapolando, podemos decir igualmente: ha emergido una nueva clase social que no concibe ya el mundo y la realidad en términos de movimientos cíclicos, periódicos o regulares. Dicho epistemológicamente, que ya no requiere única y fundamentalmente del cálculo y la estadística normal. Castells confiesa no tener un nombre para esta nueva clase social. Se trata de una nueva clase social que ve un tipo de movimiento que la burguesía jamás había visto: movimientos y dinámicas de eventos raros.

\section{Complejidad de los eventos raros}

En el lenguaje del estudio de los sistemas de complejidad creciente siempre aparecieron, desde el comienzo, características y propiedades tales como: emergencia, autoorganización, no-linealidad, bifurcaciones, y varios más. Sin embargo, en el marco del estudio sobre los fenómenos raros, cabe decir con propiedad que son otros los conceptos y atributos propios de un sistema complejo; me refiero, notablemente a: intersticios, comisuras, pliegues, vetas, moteados, quiebres, en fin, justamente, rareza y sorpresa. El prestigio de aquellos conceptos se deriva principalmente de la física y la química. La extrañeza de estos otros conceptos hace que se vinculen mucho más a metáforas y símiles.

Pues bien, cabe sintetizar estas propiedades con un concepto preciso: impredecibilidad. Así, quiero sostener que el estudio de la complejidad consiste exactamente en el estudio de fenómenos, comportamientos y sistemas impredecibles. Algo que nunca se ha puesto explícitamente de manifiesto en la bibliografía especializada sobre el tema. Más radicalmente, la complejidad consiste en el estudio de los fenómenos raros, extraños, extremos, en fin, no-conocidos.

Quisiera destacar cuatro rasgos epistemológicos que ponen de manifiesta la complejidad de los eventos raros. Estos son:

Ver lo no visto. De manera atávica, la inmensa mayoría de las personas reducen lo nuevo que ven a lo ya visto y lo ya conocido. En el marco de los estudios sobre percepción y sobre teoría de la decisión racional es lo que se conoce como "decisión por experiencia", un rasgo que se asemeja mucho a irracionalidad. La filosofía fenomenológica expresa esto en los siguientes términos: el conflicto estriba en si vemos lo que conocemos o si conocemos lo que vemos. La complejidad del evento raro consiste en conocer lo que vemos.

Pensar lo impensado. Nadie piensa bien si no piensa en todas las posibilidades. Esta es una regla de la más sana lógica y teoría de la racionalidad. Ahora bien, pensar en todas las posibilidades implica, necesariamente, considerar incluso lo imposible mismo. Pues bien, los eventos raros son, por definición, 
acontecimientos que no cabía pensar antes de que ocurrieran. En este marco, es un imperativo al mismo tempo epistemológico y ético pensar las posibilidades y lo imposible mismo, puesto que bien puede llegar a suceder que una excepción -positiva o negativa- pueda tener lugar.

Ver lo que (aún) no existe. La cultura, la ciencia y la historia que vivimos es alta y crecientemente contraintuitiva. Este es el rasgo perceptual más determinante de la sociedad de la información, la sociedad del conocimiento y la sociedad de redes. Debe ser posible ver lo que (aún) no existe, precisamente debido a que aún no ha llegado, o no se encuentra todavía entre nosotros. En el parágrafo cinco volveremos sobre esta idea, pero por lo pronto debe quedar en claro que la complejidad de los eventos raros es directamente proporcional a la capacidad de la imaginación y/o de las simulaciones que podamos llevar a cabo.

Lo posible, e incluso lo imposible mismo. La ciencia normal consiste en la preocupación por el estudio de lo real. La ciencia normal es ciencia de la realidad. Pues bien, la preocupación por los eventos raros -o lo que es equivalente, el estudio de las ciencias de la complejidad-es, por el contrario, la preocupación por lo posible mismo. En este marco, la realidad queda circunscrita como un subcomponente de un conjunto más amplio que lo comprende y lo hace posible: lo posible, justamente. Las ciencias de la complejidad son ciencias de posibilidades, más que de factualidades. Los eventos raros no son necesariamente reales: mejor aún, son siempre esencialmente posibles. Por ello mismo la exigencia epistémica y moral por comprenderlos y explicarlos: "We will not understand how people respond to rare but high-consequence events unless we understand the psychology and rationality of people's decisions from experience" (Hetwig 2012:289).

En otras palabras, la dificultad de los eventos raros es que no solamente son inesperados, sino, adicionalmente, no obedecen a los patrones habituales de comportamiento de un fenómeno determinado. Es aproximadamente como con los terremotos: se sabe que existen, se saben que sucederán, pero no se puede predecir en absoluto cuándo o cómo o con qué intensidad van a tener lugar.

En ciencia en general -como por lo demás también en literatura y en poesía, por ejemplo-, cuando las herramientas disponibles, el conocimiento heredado y los lenguajes comunes resultan insuficientes para comprender un fenómeno, se produce un verdadero acto de creación: se crean nuevas matemáticas, se crean nuevos enfoques, en fin, se crean nuevos métodos y lenguajes. Pues bien, esto justamente es lo que sucede a propósito de los eventos raros. Son los problemas los que generan la innovación en el pensamiento y el conocimiento. Y los eventos raros constituyen una complejización de la comprensión habitual del universo y la realidad, que demanda, consiguientemente, de imaginación, creatividad y aprendizaje. Pues bien, de manera puntual estas características consisten en los cuatro rasgos mencionados, a saber: el desafío de ver lo no visto, pensar lo impensado, ver incluso lo que (aún) no existe, y abocarnos al estudio, comprensión y explicación de lo posible y lo imposible mismo antes que y por encima de lo real mismo.

La consecuencia no puede ser menos sorprendente y contraintuitiva: conocer la realidad resulta insuficiente, aunque sea necesario, puesto que los eventos raros son aquellos que asaltan a lo real mismo y no cabe ser anticipados desde la dimensión puramente factual. La ciencia se alimenta de imaginación, aun cuando ingiera siempre, necesariamente observaciones y descripciones de la realidad. 


\section{4. ¿Qué es un evento raro?}

Por definición, un evento raro es contra-intuitivo, y no se ubica, en absoluto en los marcos de la estadística normal. Manifiestamente, no entra en las distribuciones normales, y habitualmente usurpa la memoria puesto que su carácter súbito e irrepetible hace que no se adecue a los patrones, modos y contenidos de la memoria humana.

Los eventos raros son imprevistos, rompen los moldes y patrones habituales, y rompen la cotidianidad en cualquier acepción de la palabra. En otras palabras, los acontecimientos extremos no se explican tanto en función de la necesidad, sino de quiebres, rupturas, discontinuidades y tiempos y espacios discretos. Más exactamente, pensar en eventos raros equivale a pensar en sistemas discretos, y en absoluto en sistemas continuos.

Se produce aquí una inflexión determinante con respecto a la cultura tradicional: "Rare events, typically low in occurrence possibility while extremely large (or extremely small) in amplitude, usually have the potential to cause giant effects. Unfortunately, it is rather difficult to understand the mechanism behind these behaviors. In each of these systems, too many factors nonlinearly interact with each other, leading their outputs to be erratic, unstable, or chaotic" (Zhao, Shang, Lin 2016: 132).

Hay fenómenos que no encajan en los marcos de lo-ya-visto y lo ya-conocido. Ni siquiera en los de lo-yapensado, o imaginado. Literalmente, estos son los acontecimientos que dan qué pensar.

Naturalmente, un evento raro no tiene por qué ser siempre necesariamente negativo. Existen también numerosos eventos raros que son magníficamente sorpresivos por lo positivo o favorable que resultan en un momento determinado. En verdad, la historia del mundo, y acaso del universo mismo está definido por numerosos eventos raros, esto es, excepcionales; y por tanto irrepetibles.

Así, por ejemplo, la vida, tal y como la conocemos surgió una sola vez en el universo y no ha vuelto a repetirse, hasta donde disponemos de información. Asimismo, el paso de una civilización a la siguiente ha sido un acontecimiento único e irrepetible. Una sola vez nació el método científico, y una sola vez han sucedido revoluciones como la francesa de 1789, la rusa de 1917 o la cubana de 1959, por ejemplo. Jamás volverán a repetirse revoluciones como éstas en la historia de la humanidad.

De la misma manera, una sola vez hubo un compositor como J. S. Bach, no volverá a haber una segunda Mme. Curie, o las atrocidades de la segunda guerra mundial son eventos singulares. A nivel individual, asimismo, cada quien ha conocido los avatares propios de una forma que para quien han sido únicos, y su vida no sería jamás la misma si no hubiera tenido esas experiencias. Becas, viajes, amores, sufrimientos, y tantas otras experiencias, únicas y excepcionales hacen de la biografía de cada quien un acontecimiento irrepetible.

Consiguientemente, los acontecimientos raros tienen la característica de que, epistemológica o cognitivamente, admiten una pluralidad de perspectivas e interpretaciones. Este rasgo se encuentra en las antípodas de los fenómenos normales y regulares, sujetos a leyes y constantes, los cuales son susceptibles de una diversidad bastante más restringida de lectura y explicación. De manera sencilla: cuanto menor sea la probabilidad de que un evento ocurra nuevamente, tanto mayor será la probabilidad de que sea considerado como raro. Precisamente en este sentido, un evento raro gatilla la complejidad del mundo y 
la naturaleza por cuanto sienta las bases para diferentes visiones y significados del mundo, la realidad y la sociedad.

De forma reciente, los acontecimientos extremos han sido llamados por un autor como cisnes negros. Pues bien, desde este punto de vista, puede decirse que toda la historia y la cultura, la ciencia y la filosofía tradicionales ha consistido de forma genérica en buscar cisnes blancos. La razón es elemental: solo existen cisnes blancos, y buscar cisnes de otros colores se revela, a la luz de esa perspectiva, como algo irracional.

En contraste, queremos sostener la idea de que la innovación, el descubrimiento científico, el pensamiento creativo consisten justamente en la tarea por tematizar y descubrir cisnes negros; esto es, acontecimientos altamente improbables. Aquí la historia de la ciencia presenta argumentos contundentes. En efecto, como lo ilustra la historia y la filosofía de la ciencia, ningún investigador ha descubierto nada en la línea de investigación en la que venía trabajando. Por el contrario, los descubrimientos científicos y tecnológicos, por ejemplo, siempre han tenido lugar: a) en las proximidades en las que venía trabajando un investigador; y b) por casualidad. Los ejemplos en ámbitos como la química y la ingeniería, la física y las matemáticas, la genética o la historia de las técnicas es prolífica al respecto. Como bien lo sostuviera Heráclito: "Si no se espera lo inesperado, no se lo hallará, dado lo inhallable y difícil de acceder que es" (2009: 22 B 18).

\section{Explicando los eventos raros}

La alta ocurrencia de eventos raros conduce a la elaboración de importantes bases de datos sobre acontecimientos extremos en numerosos dominios. Pero a fin de elaborar estas bases de datos se impone antes el muestreo de eventos raros (rare event sampling, en inglés). Diversos métodos y técnicas son subsecuentemente desarrollados. Se trata, esencialmente, del recurso a modelos de simulación computacionales mediante simulación de fuerza-bruta.

Se impone aquí una observación importante, aunque difícil. Análogamente a como la inmensa mayoría de complejólogos trabajan la complejidad con métodos analíticos, asimismo la mayoría de quienes investigan eventos raros los reducen a técnicas y métodos que les suministran tranquilidad y seguridad pero que son inocuos epistemológicamente. Los complejólogos, en su mayoría, incorporan métodos numéricos, parametrización, constantes de Lyapunov, creación de nuevos y distintos algoritmos, sistemas dinámicos y dinámica de sistemas, linealización y varios más. Análogamente, en el estudio de los eventos raros los métodos bayesianos (análisis bayesiano, redes bayesianas, etc.), métodos de confiabilidad estructural (llamados en inglés structural relaiability methods, SRM), técnicas de muestreo, y aproximación de muestreo promedios (en inglés: simple average approximation, SAA), algoritmos mejorados y otros son generalizados en el estudio y explicación de eventos raros. Pues bien, el empleo de herramientas conocidas y habituales es, desde el punto de vista heurístico, trivial, y todo ello consiste simple y llanamente en reducir la novedad y la sorpresa a métodos ya conocidos, a técnicas que se dominan, en fin, al conocimiento normal circulante en la comunidad científica o académica. Dicho en términos puntuales: todo ello consiste en conocer el evento raro, pero no en pensarlo. Una distinción básica en filosofía y epistemología, por decir lo menos.

En contraste con lo anterior, quiero sugerir cinco modos de comprender y de explicar a los eventos raros. Estos son (el orden no importa): 
La teoría del valor extremo. La estadística misma suministra una teoría propicia para la comprensión de los eventos raros: la teoría del valor extremo. Difícil y técnica matemáticamente, se trata de una teoría útil en el estudio de fenómenos estocásticos.

La contingencia. Quizás nadie como S. J. Gould ha puesto de relieve el papel que la contingencia juega en la evolución del mundo y de la vida. Desde luego que la ciencia normal sabe de contingencias, pero las normaliza. Aquí, el reto consiste en comprender el papel positivo o constructivo que la contingencia desempeña en la historia del universo y de la vida.

Las ciencias de la complejidad. Un campo amplio y en desarrollo, las ciencias de la complejidad suministran, dicho de manera puntual, dos herramientas: las distribuciones de leyes de potencia y el uso de la simulación. Aquí, resulta fundamental distinguir que una cosa es el modelamiento, y otra muy distinta la simulación. Los fenómenos lineales pueden modelarse; solo los sistemas complejos pueden simularse.

Las lógicas no-clásicas. Un capítulo en proceso de crecimiento en la historia del conocimiento, las lógicas no-clásicas (LNCS) ofrecen varias posibilidades para la comprensión de los acontecimientos extremos. Notablemente, la lógica epistémica, la lógica multimodal, y la lógica polivalente resultan de gran utilidad para la investigación sobre los eventos extremos. Una ampliación de esta idea sería el objeto de otro texto aparte.

La lógica de contrafácticos. Un lugar aparte merece la lógica de contrafácticos dado que su principal fortaleza consiste en el uso de la imaginación como heurística. La negación de los hechos primarios y la asunción de posibilidades inexploradas abre las puertas para eventos singulares e introduce al investigador en los dominios propios de las pompas de intuición y los experimentos mentales, condiciones indispensables para pensar lo impensado.

Un evento raro no puede ser explicado más que a condición de cambiar las estructuras cognitivas habituales y abrirse a dimensiones hasta entonces no consideradas (por cualesquiera motivos). Manifiestamente, se trata de acontecimientos que desafían enormemente al conocimiento y que demandan, a fin de poder anticiparlos, la máxima imaginación, métodos y técnicas concomitantes. Cuando la realidad nos desborda, queda la imaginación como recurso. Cuando está preparada y formada, cuando está nutrida de excelente formación y educación, la imaginación puede alcanzar estadios ignotos e inopinados.

Al fin y al cabo, explicar lo novedoso, distinto y singular demanda, por definición, creatividad. Una expresión en inglés afortunada: ser/pensar/vivir como outlier. Esto es, todo lo contrario a pensar y vivir en términos algorítmicos, para decirlo de manera puntual y directa. Un fantástico desafío práctico y epistemológico.

\section{Conclusiones}

Si en los comienzos de la modernidad sostenía Galileo que el lenguaje en el que habla la naturaleza son las matemáticas, es posible decir que hoy en día el lenguaje de las ciencias (esto es, en el que se comunican las ciencias entre sí, y en el que explican y comprenden el mundo) es la estadística. Se trata de la comprensión del mundo, la naturaleza y la realidad en términos de distribuciones de probabilidades. Pues bien, más exactamente, el mundo ha dejado de ser un fenómeno regular, periódico y cíclico y hemos hecho el aprendizaje de que los más apasionantes acontecimientos son eventos raros. 
El estudio, comprensión y explicación de los eventos demanda una nueva estructura mental (mindset). Digámoslo de manera puntual: una nueva epistemología (ciencia y filosofía), o distinta a la disponible en cada momento.

Los problemas y los retos, los desafíos y las apuestas constituyen los verdaderos nutrientes del pensamiento. Visto en perspectiva histórica, la humanidad no había visto anteriormente eventos raros. El concepto y el problema es perfectamente contemporáneo. Consiguientemente, nuevas herramientas, métodos, aproximaciones y técnicas se están desarrollando con el fin de comprender, explicar, y entonces hacer anticipaciones de eventos raros.

Esta clase de fenómenos, comportamientos y fenómenos comportan la complejidad del mundo y la realidad, pero entonces también, paralelamente, del pensar y conocer. A título exhortativo, debería ser posible "conocer" los eventos extremos antes de que sucedan. La simulación y el buen ejercicio de la imaginación constituyen herramientas provechosas paralelas y emergen como heurísticas sin iguales. He aquí un tema y un problema abiertos por definición. Pues bien, la condición para ver y trabajar con sistemas abiertos consiste en una apertura de mente; algo que, sin embargo, se dice fácilmente, pero es difícil de llevar a cabo.

Digamos, a título de conclusión que los eventos raros definen, frontalmente y sin tecnicismos, la complejidad misma de la naturaleza y la sociedad. Eventos raros, acontecimientos extremos, fenómenos excepcionales: todos ellos nos permiten comprender que el mundo y la realidad son moteados, con matices, con vetas, en fin, irregulares. Un auténtico desafío a la idea de un mundo estable, en equilibrio, con ciclos periódicos, sujeto a leyes incambiables.

\section{Bibliografía}

Heráclito. 2009. Fragmentos. En: AA.VV. Filósofos presocráticos. Madrid: Editorial Gredos.

Hertwig, R. 2012. The psychology and rationality of decisions from experience. Synthese 187(1): 269-292. doi: 10.1007/s11229-011-0024-4

Zhao, X; Shang, P. and Lin, A. 2016. Universal and non-universal properties of recurrence intervals of rare events. Physica A: Statistical Mechanics and its Applications 448: 132-143. doi:

10.1016/j.physa.2015.12.082

Recibido el 30 May 2016

Aceptado el 14 Jul 2016 